\title{
Experimental Study About Water Saturation Influence on Changes in Reservoirs Petrophysical Properties
}

\author{
Sudad Hameed AL-OBAIDI ${ }^{1, *}$, Victoria SMIRNOV ${ }^{1}$ and Hiba Hussein ALWAN ${ }^{2}$ \\ ${ }^{I}$ Department of Petroleum Engineering, Mining Institute, SPB, Russia \\ ${ }^{2}$ Department of Petroleum Engineering, Knowledge University, Erbil, Iraq
}

('Corresponding author's e-mail: sudad.hameed@koyauniversity.org)

Received: 21 March 2021, Revised: 22 April 2021, Accepted: 29 April 2021

\begin{abstract}
Experimental determination of the physical properties of rocks under conditions simulating in situ reservoir conditions is of great importance both for the calculation of reserves and for the interpretation of well logging data. In addition, it is also important for the preparation of hydrocarbon field development projects. The study of the processes of changes in the petrophysical properties of the reservoir under controlled conditions allows not only to determine their reliability but also to evaluate the dynamics of these changes depending on the temperature and pressure conditions of the reservoir and the water saturation of the rocks.

In this work, an evaluation of the dependence of the physical properties of hydrocarbon reservoirs on their water saturation $\left(\mathrm{S}_{\mathrm{w}}\right)$ was carried out. Residual water saturation $\left(\mathrm{S}_{\mathrm{wr}}\right)$ was created in the rocks and the properties of these rocks were compared at the states of partial $(25 \%)$ and complete water saturation $(100 \%)$. The changes in petrophysical parameters of partially water saturated rocks during the increase in effective pressure were studied and estimates of these changes were obtained. The results showed that when the effective pressure is increased, the $S_{\mathrm{wr}}$ increases by an average of $6 \%$ compared to atmospheric conditions. This is accompanied by an increase in the velocity of longitudinal (by $51.9 \%$ on average) and lateral waves (by $37.1 \%$ on average). As residual water saturation increases, effective permeability decreases for both standard and reservoir conditions, with, gas permeability decreasing for both dry samples (by $23 \%$ on average) and samples with residual water saturation (effective permeability decreases by $27 \%$ on average).
\end{abstract}

Keywords: Petrophysical properties, Reservoir simulation, Residual water saturation, Effective permeability, Reservoir, Standard conditions

\section{Introduction}

During the development of reservoirs, significant changes occur in the hydrodynamic regime and stress-strain state that affect the physical and mechanical properties of the rocks and determine the operating characteristics of the reservoir strata.

Petrophysics determine rock samples' physical properties under standard (atmospheric) conditions and under conditions that simulate reservoir conditions [1,2]. Petrophysicists usually explain variations in physical properties of samples of the same rock in terms of changes in general mineral and chemical composition and structural and textural features $[3,4]$. Changes in physical properties of hydrocarbon reservoirs are mainly studied by determining physical properties (permeability, porosity, elastic, electrical, and deformation strength) under the standard conditions and in physical modeling of reservoir conditions and processes.

When oil fields are developed, a significant amount of oil (up to $60-80 \%$ ) remains unrecovered $[5,6]$. The distribution of hydrocarbon fluids and water in the pore space and the way they flow largely 
depend on the petrophysical properties of the rocks. During oil and gas field development, the reservoir's water content often increases, leading to a change in physical properties [7,8]. For example, several studies have shown that moisture can lead to a drastic reduction in strength and an increase in deformability in some sedimentary rocks [9-11]. This study focused on the effect of water saturation of hydrocarbon core samples on the changes in physical properties of these samples, both at standard and reservoir conditions.

\section{Materials and methods}

To assess the effectiveness of water saturation on the physical properties of the reservoir, comparisons were made between the petrophysical properties of reservoir core samples in which the pore space is fully saturated with the reservoir fluid model and samples with residual water saturation.

Samples of sandstones and siltstones of hydrocarbon reservoirs located at depths from 2,742 to 2,900 $\mathrm{m}$ were studied. The range of changes at standard conditions for porosity was from 2.9 to $33.4 \%$, for gas permeability - from 2.91 to $1,557 \mathrm{md}$. Two hundred and forty samples were saturated with a model of formation water; gas permeability was measured on 40 samples.

The residual water saturation of the rock, $\mathrm{S}_{\mathrm{wr}}$, determined by the direct method (from the core taken during drilling with an anhydrous solution) or by indirect methods (centrifugation, with semipermeable membranes, etc.), should be corrected taking into account the reservoir conditions $[12,13]$. If we assume that the main change in $\mathrm{S}_{\mathrm{wr}}$ occurs due to the deformation of the pore space of the rock during core drilling due to the reduction of the effective stress, then we can write:

$S_{w r(f m)}=S_{w r(s c)} \frac{\varphi_{s c}}{\varphi_{f m}}$

Where $S_{\mathrm{wr}(\mathrm{fm})}$ - residual water saturation (irreducible) corresponding to reservoir conditions; $\mathrm{S}_{\mathrm{wr}(\mathrm{sc})}$ is residual water saturation determined directly or by one of the indirect methods at standard conditions (laboratory conditions); $\varphi_{\mathrm{sc}}, \varphi_{\mathrm{fm}}$ - formation porosity, respectively, at standard conditions and at the depth of the deposit (formation conditions).

Residual water saturation was generated in the samples by centrifugation $[14,15]$. The centrifugation method is based on the action of centrifugal forces on the sample, which occurs during the rotation of the core sample in the centrifuge. Initially, as the speed of the centrifuge rotor increases, water is forced out of the large pores. As the rotor speed continues to increase, water is forced out of the smaller pores. At some point, increasing the number of revolutions no longer affects the amount of water remaining in the pores. This water is referred to as residual water [16].

Methods for determining the physical properties of the rock sample under atmospheric conditions and conditions of reservoir simulation are described in detail in the scientific literature $[17,18]$. These methods have been used to determine changes in pore volume, pore compressibility, formation porosity and bulk density (in reservoir conditions simulation), elastic wave velocity, electrical resistivity and formation factor.

\section{Results and discussion}

The measured and calculated data, namely porosity, bulk density, formation resistivity $\left(\mathrm{R}_{\mathrm{t}}\right)$, formation factor $(F)$, elastic wave propagation velocity and $S_{w r}$ of the samples, were collected in a summary table for the boreholes according to the measurement conditions (reservoir and atmospheric). Since the primary values of $\mathrm{S}_{\mathrm{wr}}$ were obtained under atmospheric conditions, it was necessary to recalculate them for reservoir conditions (Figure 1) using Eq. (1). The recalculation is based on the fact that the volume of water in the pore space does not change and the porosity decreases slightly during the transition from standard to reservoir conditions $[19,20]$. 


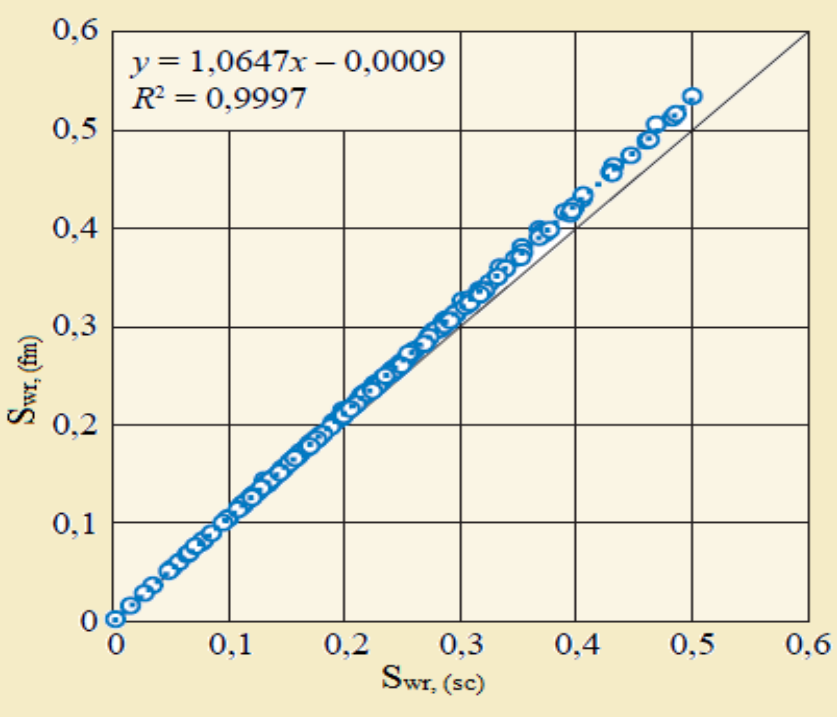

Figure 1 Correlation of residual water saturation at atmospheric (standard) and reservoir conditions: $\mathrm{R}^{2}-$ correlation coefficient.

It is well known that $\mathrm{R}_{\mathrm{t}}$ depends strongly on the water content in the pore space. Natural solutions filling pores and cracks conduct electric current much better than the mineral matrix of rocks, so as the moisture content in the rock increases, the electrical resistivity decreases significantly [21,22]. Figure 2 shows a logical decrease in $\mathrm{R}_{t}$ with increasing water saturation: The correlation coefficient for reservoir conditions $\left(R^{2}=0.71\right)$ is higher than for standard conditions $\left(R^{2}=0.46\right)$, possibly due to closure of some cracks and an increase in the role of intergranular porosity [23]. The $\mathrm{R}^{2}$ values for reservoir conditions are quite high. Thus, it can be seen that the process of reservoir water-cut (increase in $S_{w r}$ ), even with the continuation of effective pressure, leads to a decrease in resistivity.

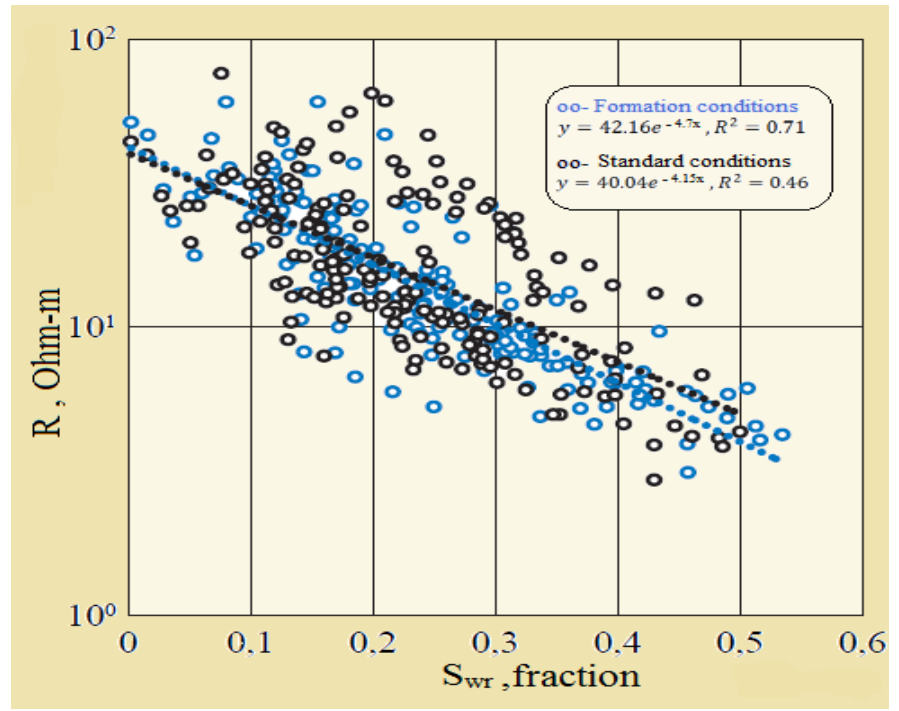

Figure 2 Effect of residual water saturation on resistivity at atmospheric and reservoir conditions. 
This study also compared the dependence of resistivity on formation porosity under reservoir conditions, both at residual and $100 \%$ water saturation (Figure 3). In this case, a good correlation coefficient $\left(\mathrm{R}^{2}=0.90\right)$ was found only for resistivity of samples at $100 \%$ water saturation, and for resistivity of samples at partial water saturation, the accuracy of the correlation coefficient was low $\left(\mathrm{R}^{2}=\right.$ 0.09). This can be attributed to the incomplete filling of the pore space with water and the complex structure of the conduction channels. Figure 3 once again confirms the above assumption that the process of formation water-cut (increase in $\mathrm{S}_{\mathrm{wr}}$ ), even if the effective pressure persists, leads to a decrease in resistivity. The performed tests show that under the conditions simulating the reservoir, with an increase in the average values of water saturation from residual $(0.25)$ to full (1.00), the average value of resistivity decreases from 19.6 to $2.24 \mathrm{ohm}-\mathrm{m}(88.6 \%)$.

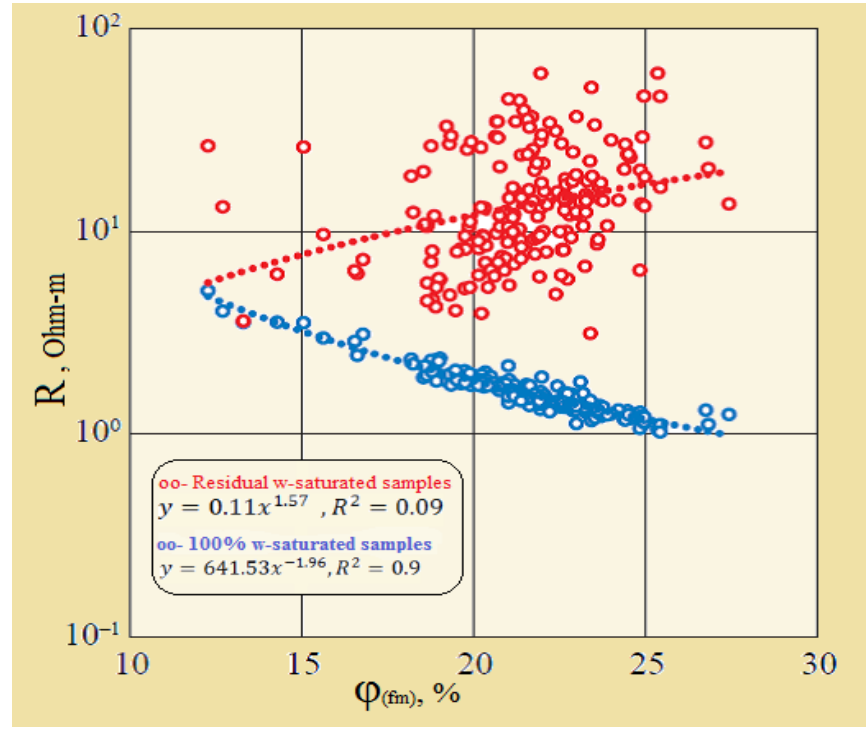

Figure 3 Dependence of resistivity at reservoir conditions on porosity at $100 \%$ and partial water saturation.

A rock containing oil and/or gas has a higher resistivity than the same rock saturated only with formation water, and the higher the water saturation of the pores, the lower the resistivity of the rock $[24,25]$. This relationship between the resistivity of the rock and its water saturation, expressed by the formation factor, is often used to distinguish hydrocarbon-bearing zones [26,27].

Figure 4 shows the effect of residual water saturation on the formation factor at standard and reservoir conditions. The formation factor generally indicates by how many times the resistivity of a partially water-saturated rock increases compared to its resistivity when the pore volume is fully saturated with water $[28,29]$. Moreover, since the volume of water in the pores of the rock samples does not change during the transition from the standard conditions to the reservoir conditions, the dependences of the formation factor on water saturation turn out to be close in this case. The slight discrepancy is due to changes in the structure of the pore space under the influence of the reservoir conditions. 


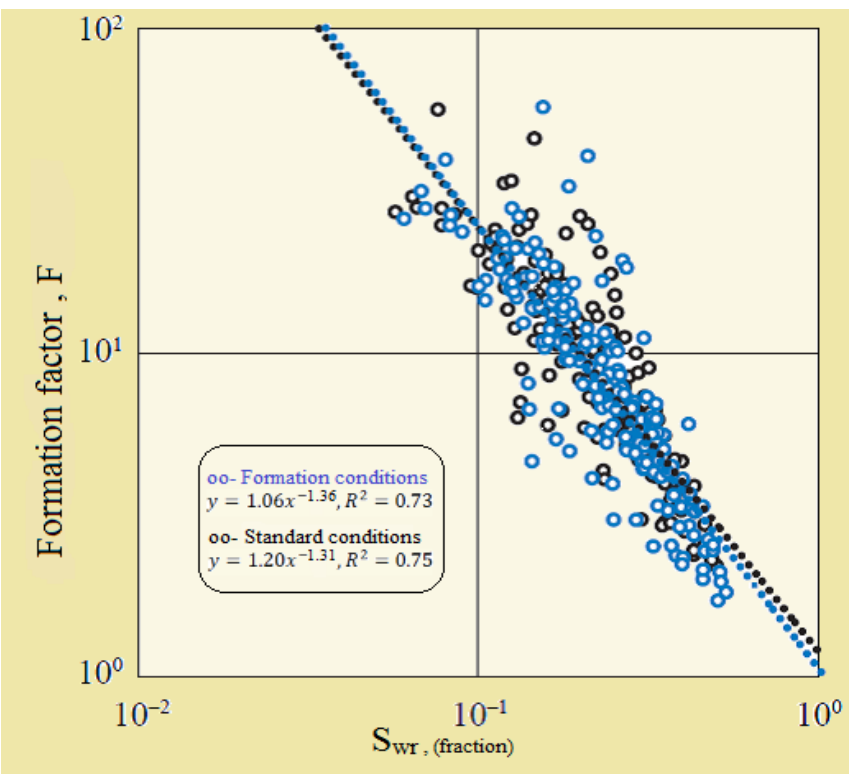

Figure 4 Effect of residual water saturation on the formation factor at atmospheric (standard) and reservoir conditions.

The study also considered the influence of water saturation on the propagation velocity of longitudinal and lateral elastic waves (Figures 5 and 6). The partial presence of formation water in the pore space should mean that the velocity of the P-wave (longitudinal wave) in a rock sample with residual water saturation will be lower than in the same sample whose pore space is, however, completely saturated with formation water (see Figure 5).

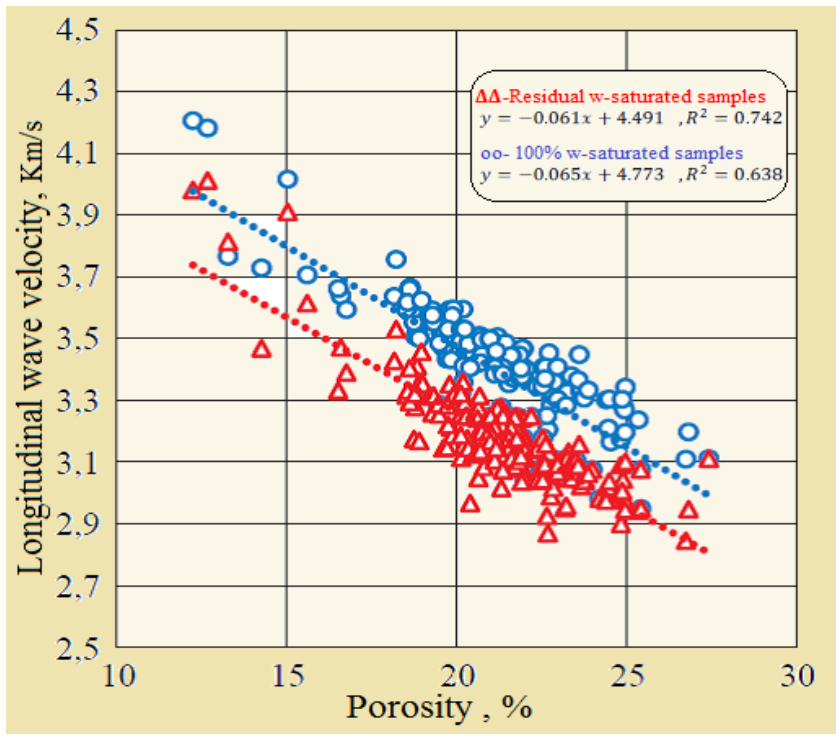

Figure 5 Dependence of the propagation velocity of a longitudinal wave on porosity at residual and 100 $\%$ water saturation of rock samples under conditions simulating reservoir. 
In Figure 5, it can be seen that the velocity of the longitudinal wave (P-wave) decreases with an increase in the porosity of the samples at both residual and $100 \%$ water saturation. The graph of the change in the velocity of the longitudinal wave for the samples with residual water saturation is slightly lower on the coordinate plane than the same graph of the samples with $100 \%$ water saturation. This is due to the fact that part of the pore space is filled with air and the propagation velocity of elastic waves in air is known to be lower than in water [30,31].

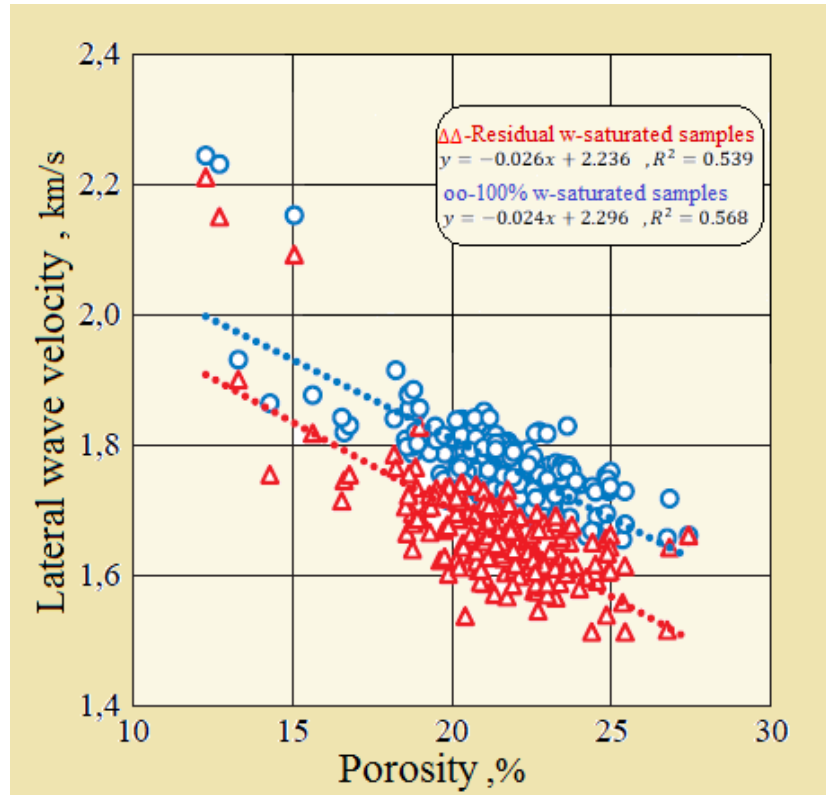

Figure 6 Dependence of shear wave propagation velocity on porosity at residual and $100 \%$ water saturation of rock samples under conditions simulating a reservoir.

The velocity of propagation of the lateral wave (s-wave) is higher in reservoir conditions, as in a longitudinal wave, than in standard conditions. However, the reliability of the correlation coefficient due to a linear dependence (see Figure 6) is much lower than for a longitudinal wave (see Figure 5). This is probably because shear waves (laterals) do not propagate in the fluid and the presence of partial water saturation in rock samples complicates the pathways (probably due to swelling of the clay component) for shear wave propagation compared to the pathways in samples with $100 \%$ water saturation.

The tests carried out have shown that under the conditions simulating the reservoir, with an increase in the average values of water saturation from a residual level of 0.25 to 1.00 , the velocities of longitudinal and lateral waves, respectively, increase from 3.21 to $3.42 \mathrm{~km} / \mathrm{s}(6.54 \%)$ and from 1.68 to $2.08 \mathrm{~km} / \mathrm{s}(23.8 \%)$. Figures 5 and $\mathbf{6}$ suggest that the process of water-cut in the reservoir (increase in $\mathrm{S}_{\mathrm{wr}}$ ) leads to a decrease in the propagation velocities of elastic longitudinal and lateral (shear) waves even at constant effective pressure.

Most interesting is the study of the effect of $S_{w r}$ on the effective permeability of rock samples, which directly affects well production rates [32-34]. Figure 7 shows the dependence of effective permeability $\left(\mathrm{K}_{\mathrm{eff}}\right)$ on $\mathrm{S}_{\mathrm{wr}}$ at reservoir and standard conditions for all samples, without dividing them into classes. 


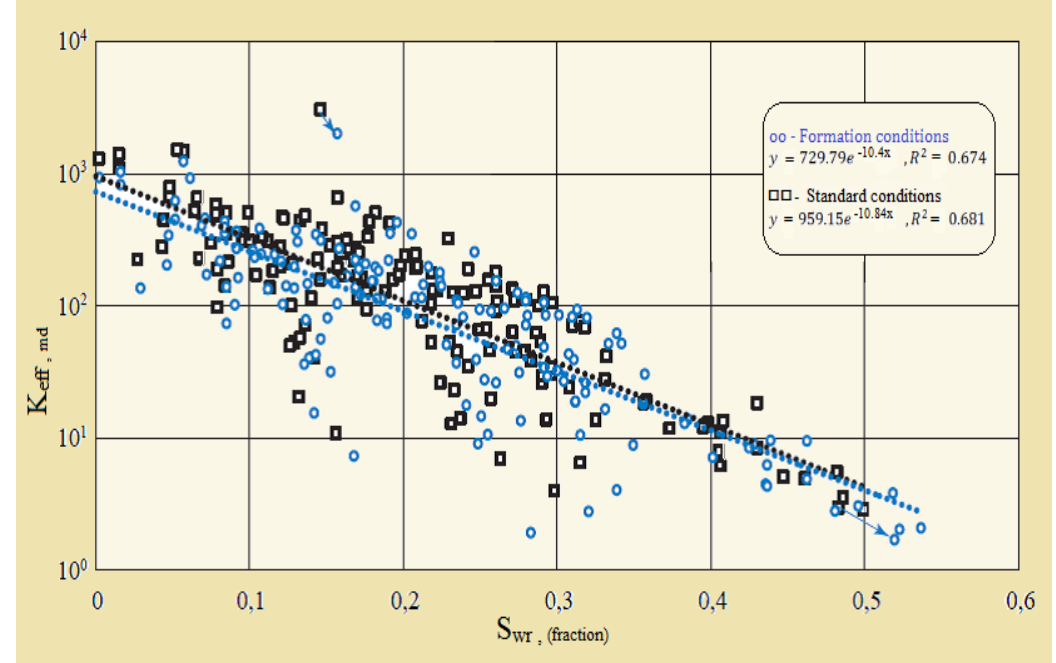

Figure 7 Dependence of effective permeability of samples on their residual water saturation at atmospheric and reservoir conditions.

Hydrocarbon reservoirs can be grouped by class according to the average values of their effective permeability [35,36]. Thus, using the same concept as in Figure 7, dependencies can be built to predict the change in effective permeability by class. Analysing Figure 7, it can be concluded that as the water saturation of the rock samples increases from 0 to $54.0 \%$, their effective gas permeability will decrease under both standard and reservoir conditions and therefore there will be a transition from higher to lower permeability classes [37]. In fairness, it should be noted that this graph reflects the influence not only of residual water saturation, but also of porosity and structure of the pore space on permeability.

The changes in permeability of dry samples $(K)$ and samples with partial water saturation $\left(K_{\text {eff }}\right)$, relative to their permeability under standard conditions $(K=1)$, were studied in the process of increasing the effective pressure. The change of $S_{w r}$ in this process was also followed. The graphs in Figure 8 show that with an increase in effective pressure the permeability of the rock decreases significantly, which seems logical. 


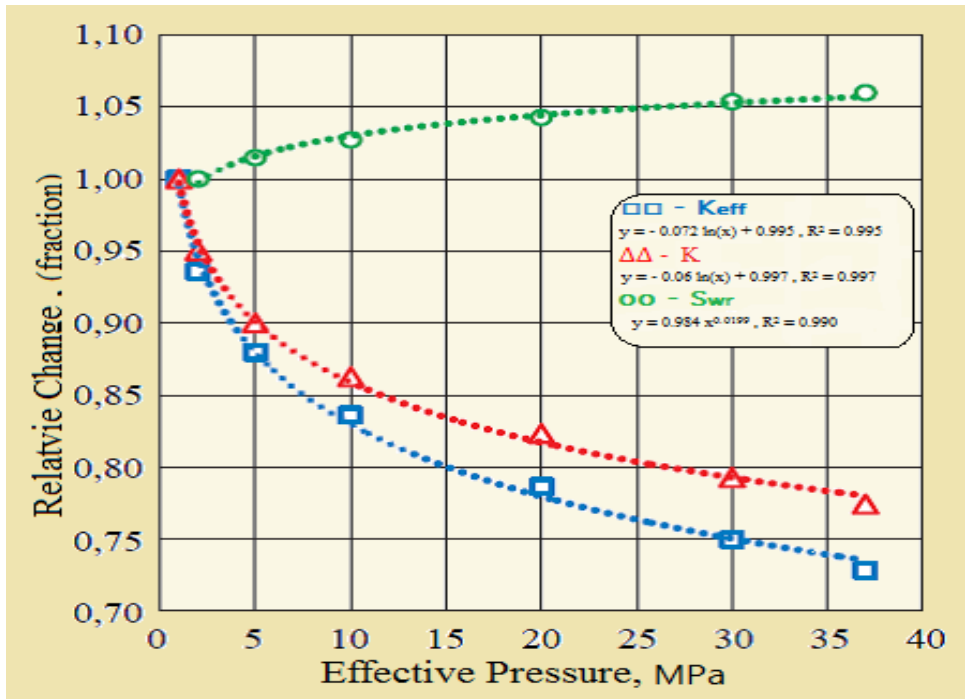

Figure 8 Change in permeability and residual water saturation with increasing effective pressure.

It can also be seen that the effective gas permeability $\left(\mathrm{K}_{\mathrm{eff}}\right)$ of partially water-saturated samples decreases more than $\mathrm{K}$ for dry samples. At the maximum effective pressure, the permeability for dry and partially saturated samples decreases by 23 and $27 \%$ on average, respectively. The decrease occurs uniformly, even for different permeability values. At the same time, the $\mathrm{S}_{\mathrm{wr}}$ increases due to a decrease in pore volume with an increase in effective pressure.

\section{Conclusions}

Experimental determination of the physical properties of rocks under conditions that simulate reservoir conditions is of great importance for the calculation of reserves and the interpretation of welllogging data, as well as for the preparation of hydrocarbon field development projects.

For example, a comparison of the petrophysical parameters of samples studied under conditions simulating the reservoir showed that with an increase in the average values of $\mathrm{S}_{\mathrm{wr}}$ from the residual 25.0 $\%$ water saturation to the full $100 \%$, the average resistivity value decreases from 19.6 to $2.24 \mathrm{ohm}-\mathrm{m}$ $(88.6 \%)$ and the velocities of longitudinal and lateral (shear) waves increase from 3.21 to $3.42 \mathrm{~km} / \mathrm{s}(6.54$ $\%)$ and from 1.68 to $2.08 \mathrm{~km} / \mathrm{s}(23.8 \%)$, respectively.

The results of the study of changes in petrophysical parameters of partially water-saturated rocks in the course of increasing the effective pressure from atmospheric (standard) to reservoir conditions showed that when the effective pressure is increased, the $\mathrm{S}_{\mathrm{wr}}$ increases by an average of $6 \%$ relative to atmospheric conditions. This is accompanied by an increase in the velocity of longitudinal (by $51.9 \%$ on average) and lateral waves (by $37.1 \%$ on average).

Gas permeability decreases for both dry samples (by $23 \%$ on average) and samples with residual water saturation (effective permeability decreases by $27 \%$ on average).

These data can be used in field development planning to assess both changes in reservoir conditions and the degree of change in pay zones. In other words, the results of this work contribute to effective, more accurate implementation of hydrocarbon field development, e.g., accurate reserve estimation, enhanced oil recovery, etc.

It should be noted that additional experimental studies of samples under thermobaric conditions simulating those of the reservoir are necessary to refine the changes described by power or exponential equations. 


\section{References}

[1] VK Bust, AA Majid, JU Oletu and PF Worthington. The petrophysics of shale gas reservoirs. Pet. Geosci. 2013; 19, 91-103.

[2] MS Magid. Physical properties of mountain rocks and minerals (petrophysics). 1984, p. 455.

[3] MA Zdanov. Geology and evaluation of oil and gas. $2^{\text {nd }}$ eds. 1981, p. 453.

[4] American Petroleum Institute (API). Recommended practices for core analysis. API Publishing Services, New York, 1998.

[5] VI Smirnov and SH Al-Obaidi. Innovative methods of enhanced oil recovery. Oil Gas Res. 2008; 1, $1000 \mathrm{e} 101$.

[6] D Tiab and EC Donaldson. Theory and practice of measuring reservoir rock and fluid transport properties. Gulf Publishing Company, 1996.

[7] V Kułynycz. The influence of wettability on the petrophysical parameters of reservoir rocks. $A G H$ Drilling Oil Gas 2017; 34, 775-84.

[8] AL Endres and R Knight. The effects of pore-scale fluid distribution on the physical properties of partially saturated tight sandstones. J. Appl. Phys. 1991; 69, 1091-8.

[9] SH AL-Obaidi, M Hofmann and IP Kamensky. Changes in the physical properties of hydrocarbon reservoir as a result of an increase in the effective pressure during the development of the field. $J$. Pet. Eng. Technol. 2010; 1, 16-21.

[10] IP Kamensky, SH AL-Obaidi and FH Khalaf. Scale Effect in laboratory determination of the properties of complex carbonate reservoirs. Int. Res. J. Mod. Eng. Technol. 2020; 2, 1-6.

[11] E Sondena, F Bratteli, HP Normann and K Kolltveit. The effect of reservoir conditions and wettability on electrical resistivity. In: Proceedings of the SPE Asia-Pacific Conference, Perth, Australia. 1991.

[12] SH Al-Obaidi and AP Galkin. Dependences of reservoir oil properties on surface oil. J. Pet. Eng. Emerg. 2005; 5, 74-7.

[13] WG Anderson. Rock/oil/brine interactions and the effects of core handling on wettability. J. Pet. Technol. 1986; 38, 1125-44.

[14] M Fleury. A new technique for setting irreducible water saturation on core samples. In: Proceedings of the International Symposium of the Society of Core Analysis, Noordwijk, Natherlands. 2009.

[15] RL Slobod, A Chambers and WL Prehn. Use of centrifuge for determining connate water, residual oil, and capillary pressure curves of small core samples. J. Pet. Technol. 1951; 3, 127-34.

[16] K Katika. 2016, Rock physics of reservoir rocks with varying pore water saturation and pore water salinity. Ph. D. Dissertation. Technical University of Denmark, Denmark.

[17] DG Longeron, MJ Argaud and L Bouvier. Resistivity index and capillary pressure measurements under reservoir conditions using crude oil. In: Proceedings of the Annual Technical Conference, San Antonio, Texas. 1989.

[18] SH Al-Obaidi. Comparison of different logging techniques for porosity determination to evaluate water saturation. engrXiv Preprints 2021. http://dx.doi.org/10.31224/osf.io/fvj9u.

[19] A Poupon and J Leveaux. Evaluation of water saturation in shaly formations. In: Proceedings of the SPWLA $12^{\text {th }}$ Annual Logging Symposium, Dallas, Texas. 1971, p. 1-2.

[20] WM Stoll, HA Shureqi, J Finol, SA Al-Harthy, S Oyemade, AD Kruijf, JV Wunnik, F Arkesteijn, R Bouwmeester and MJ Faber. Alkaline/surfactant/polymer flood: From the laboratory to the field. SPE Res. Eval. Eng. 2011; 14, 702-12.

[21] WG Anderson. The effects of wettability on the electrical properties of porous media. J. Pet. Technol. 1986; 38, 1371-78.

[22] GV Keller. Effect of wettability on the electrical resistivity of sands. Oil Gas J. 1953; 51, 62-5.

[23] A Settari. Reservoir compaction. J. Pet. Technol. 2002; 54, 62-9.

[24] G Mavko, T Mukerji and J Dvorkin. The rock physics handbook: Tools for seismic analysis of porous media. Cambridge University Press, 2009.

[25] SH Al-Obaidi. Improve the efficiency of the study of complex reservoirs and hydrocarbon deposits East Baghdad Field. Int. J. Sci. Technol. Res. 2016; 5, 129-31. 
[26] Schlumberger. Log interpretation principles/applications. Schlumberger Educational Services, 1998.

[27] VN Dakhnov. Geophysical methods for determination of reservoir quality and oil-gas-bearing capacity of rocks. Nedra, 1975.

[28] SH Al-Obaidi. Модификация Уравнения Арчи Для Определения Водонасыщенности Нефтяного Месторождения Восточный Багдад. OSF Preprints 2020. https://doi.org/10.31219/osf.io/tqpn5.

[29] H Lu, H Tang, M Wang, X Li, L Zhang, Q Wang, Y Zhao, F Zhao and J Liao. Pore structure characteristics and permeability prediction model in a cretaceous carbonate reservoir, North Persian Gulf Basin. Geofluids 2021; 2021, 8876679.

[30] $\mathrm{SH}$ Al-Obaidi and FH Khalaf. Acoustic logging methods in fractured and porous formations. $J$. Geol. Geophys. 2017; 6, 1000293.

[31] W Si, B Di, J Wei and Q Li. Experimental study of water saturation effect on acoustic velocity of sandstones. J. Nat. Gas Sci. Eng. 2016; 33, 37-43.

[32] WG Anderson. The effects of wettability on relative permeability. J. Pet. Technol. 1987; 39, 145368.

[33] SH AL-Obaidi, VI Smirnov and IP Kamensky. Investigation of rheological properties of heavy oil deposits. Int. J. Sci. Technol. Res. 2019; 8, 2394-7.

[34] SJ Salter and KK Mohanty. Multiphase flow in porous media. In: Proceedings of the SPE Annual Technical Conference and Exhibition, New Orleans, Louisiana. 1982.

[35] AA Hanin. Oil and gas reservoir rocks (in Russian). Publishing House Nedra, Moscow, 1965.

[36] O Falode and E Manuel. Wettability effects on capillary pressure, relative permeability, and irreducible saturation using porous plate. J. Pet. Eng. 2014; 2014, 465418.

[37] Z Li, C Du, Y Tang and X Li. Experimental and statistical investigation of reservoir properties with the effect of waterflooding treatment. ACS Omega 2020; 5, 20922-31. 